\title{
Mapping GPS Multipath: a Case Study for the Lunar Laser Ranger Timing Antenna at HartRAO
}

\author{
Cilence Munghemezulu ${ }^{1,2}$, Ludwig Combrinck ${ }^{1,2}$, Joel Botai ${ }^{1,3}$, Zinhle Mashaba ${ }^{1,4}$
}

${ }^{1}$ Department of Geography, Geoinformatics and Meteorology, Centre for Geoinformation Science, University of Pretoria, South Africa, cilence.munghemezulu@up.ac.za

${ }^{2}$ Hartebeesthoek Radio Astronomy Observatory, P O Box 443, Krugersdorp 1740, South Africa

${ }^{3}$ South African Weather Services, Pretoria, South Africa

${ }^{4}$ Geoinformatics Division, Agricultural Research Council, Pretoria, South Africa

\section{http://dx.doi.org/10.4314/sajg.v5i2.4}

\begin{abstract}
Accounting for multipath in Global Navigation Satellite Systems (GNSS) is a difficult task and an important one, especially during the pre-investigation phase for the installation of a permanent GNSS station for positioning or timing applications. Sites with a high level of multipath can cause positioning errors or timing errors resulting in the quality of GNSS products (position or timing) becoming degraded by several metres or nanoseconds. We investigate and attempt to map multipath as part of the site investigation for the installation of the timing antenna for lunar laser ranging applications at the Hartebeesthoek Radio Astronomy Observatory (HartRAO). A high-resolution wavelet power spectrum and a standard deviation parameter are used to map multipath in both the time and frequency domain as well as spatial variations on the sky plot. The high standard deviation values on the sky map are attributed to reflections due to shrubs or trees on the site, while smaller standard deviation areas are attributed to bare soil or less vegetated as this would give constant reflection over time provided the ground has constant moisture. We conclude that the site is suitable for installation of the timing antenna and that a mask of $15^{\circ}-20^{\circ}$ elevation angle will be applied to the timing antenna to minimise multipath at lower elevations.
\end{abstract}

\section{Introduction}

Multipath signals in most Global Navigation Satellite Systems (GNSS) applications are considered noise. Multipath occurs when an electromagnetic signal arrives at an antenna along an indirect path due to the deflection and reflection of the signal by nearby objects or the surface near the antenna (Larson et al., 2008). Multipath signals are caused by surrounding features such as trees or soil reflecting transmitted signals from the satellites to the antenna. Trees affect the frequencies in the L-band, the leaves and the trunk cause 
shadowing and attenuation, scattering originates from the tree branches, reflection and diffraction are caused mainly by the tree trunk (Schubert et al., 2010). Soil moisture changes affect the phase of the Signal-to-noise ratio (SNR) modulation pattern and its magnitude when a GPS satellite decends or ascends at low elevations (Zavorotny et al., 2010). The Lband signals penetrate further in dry soil as compared to wet soil (Njoku and Entekhabi, 1996).

The errors caused by multipath on GNSS data adversely affect GNSS products, these products include positioning (Closas et al., 2009), timing (Ray and Senior, 2003) and Integrated Precipitable Water Vapour (IPWV) (Tregoning et al., 1998). However, recent studies have shown that multipath can be used in the GNSS-Reflectometry (GNSS-R) technique to study soil moisture content around GNSS antennas (Larson et al., 2010; Mironov and Muzalecsjiy, 2012), snow depth estimation (Botteron et al., 2013), and in the estimation of height and moisture content of the vegetation around GNSS antennas (Wan et al., 2015). Application of GNSS-R is also important in characterising sites for geodetic applications, where accuracy is of high priority such as time transfer techniques using GNSS (Ray and Senior, 2003).

Radio signals transmitted by the GNSS satellites are also affected by the ionosphere. The ionosphere is the upper layer of the atmosphere about $60 \mathrm{~km}$ from the Earth's surface and it contains free ions and electrons that are produced by solar radiation (Ratcliffe, 1972). The ionosphere causes a delay of radio signals. The ionospheric effect can result in a delay of up to 30ns (Giffard, 1999). However, the error introduced by the ionospheric effect can be minimised by using dual-frequency receivers and applying the double differencing method (Pullen et al., 2009). The troposphere also introduces a delay of up to metres of the radio wave. The delay introduced by water vapour in the troposphere increases with a decrease in elevation angle. This delay is usually corrected by applying tropospheric delay models such as the GPT2w developed by Böhm et al. (2015).

HartRAO is currently building a Lunar Laser Ranging (LLR) station. The LLR station requires an accurate timing system, which must be steered by the Global Positioning System (GPS). The GPS satellites broadcast position and time information to the end-user. Satellites are constantly updated with accurate timing information from the ground-based network of Caesium and Hydrogen master clocks (Allan, 1980), which is referred to as time transfer and GPS plays an integral part in this regard. A geodetic observatory that requires precise accurate station time. Station time is required in order to be accurate enough (within nanoseconds of UTC) to be able to track earth-orbiting satellites or retro-reflectors located on the surface of the Moon with high accuracy.

The technique of LLR involves transmitting short laser pulses to the retro-reflectors that are located on the surface of the Moon. The round-trip time-of-flight of the laser pulses is measured using an event timer. The event timer requires a stable frequency (which can range 
from 5 to $100 \mathrm{MHz}$ ) from the local timing system (e.g. rubidium clock). The local timing system drifts slowly over time and this requires accurate time updates from GPS to be updated and monitored. The measurement data are used to support and maintain the International Terrestrial Reference Frame (ITRF), determination of the Moon's orbit, and to study geodynamics and Earth-Moon dynamics. HartRAO aims to achieve millimetre-ranging accuracy. This will require all sub-systems (these include: pointing model, timing, laser, transmit and receive path efficiencies, etc.) to be accurate, for the minimisation of station error budget to better than centimetre level. A detailed review of the LLR technique is given in Munghemezulu et al., (2016) and the references therein. In this paper, we present an experimental study, which aims to map multipath around the new site for the timing antenna for the new LLR station located at the Hartebeesthoek Radio Astronomy Observatory (HartRAO).

\section{Material and Methodology}

\subsection{Study Area and Data}

HartRAO is located in a valley of Magaliesberg mountain ranges, 50km northwest of Johannesburg. HartRAO is collocated with four instruments that are fundamental for space geodetic techniques, namely: Very Long Baseline Interferometry (VLBI), Satellite Laser Ranging (SLR), Global Navigation Satellite Systems (GNSS) antenna and Doppler Orbitography and Radio-positioning Integrated by Satellite (DORIS). Currently, the observatory is building a Lunar Laser Ranging system and a new 15 m VLBI2010 Global Observing System (VGOS) antenna (Figure 1).

The site for the timing antenna was chosen according to the following considerations: (i) the site must be close to the control room to minimise signal delay due to cable length, as this reduces the systematic errors introduced by the cable and variations of the ambient air temperature, (ii) the site must have minimal objects that can introduce multipath such as trees and reflective objects such as the LLR enclosure and the 26m VLBI antenna. However, since some of the objects cannot be removed, an observational strategy of observing above certain elevation angle has to be established to minimise the effects of multipath. Figure 2 illustrates the visibility of the satellites at the HartRAO site, the centre point represents the location of the proposed site for the timing antenna.

An experiment was carried out using a Topcon antenna and receiver (GB1000) for a period of 10 days. Observations were scheduled for days when there was a clear sky as clouds often introduce random multipath errors (Rao et al., 2013). The raw observations files were converted from Topcon Positioning System (TPS) format to the Receiver Independent Exchange (RINEX) format as recommended by Gurtner (2007). 


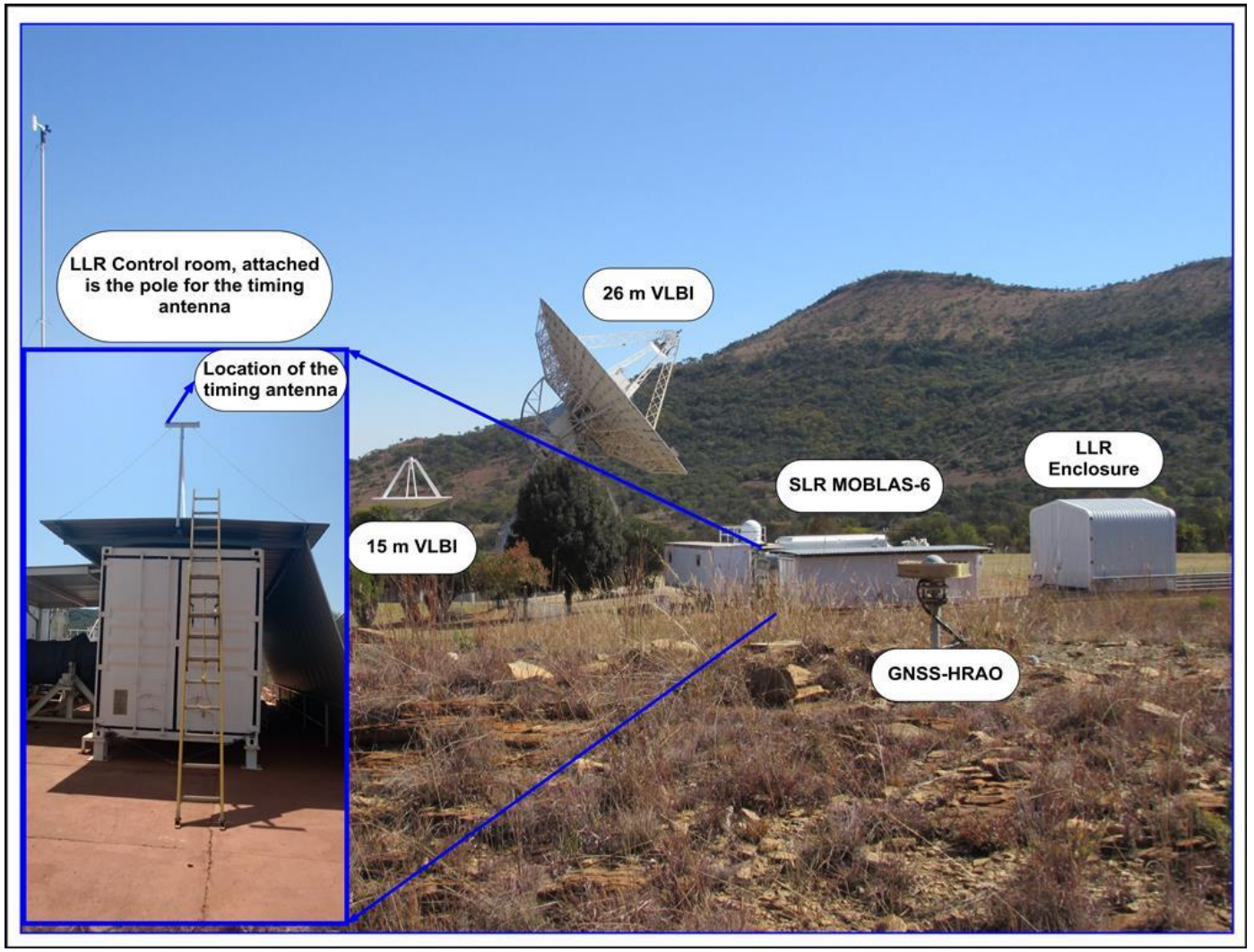

Figure 1. Space geodetic techniques collocated at HartRAO. The timing antenna will be mounted on top of the control room. The continuous operating GNSS station (HRAO) is important as it acts as a base/control station in solving positions of other points

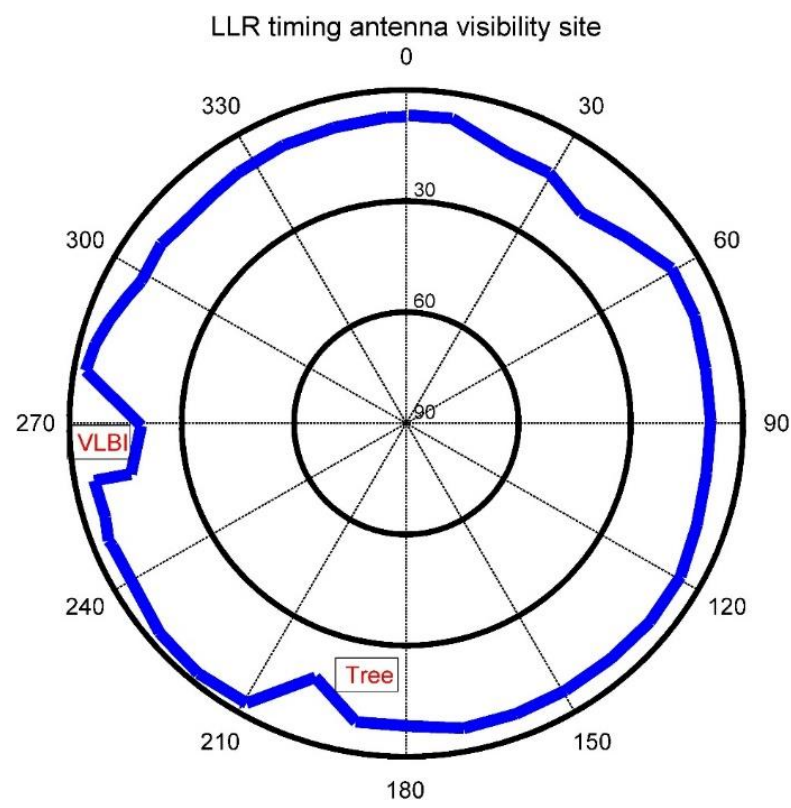

Figure 2. Horizon profile of the proposed timing antenna at HartRAO 


\subsection{Methodology}

A method for computing pseudo-range multipath errors (MP1 and MP2) was devised to assess the level of multipath in the vicinity of the GNSS antenna by making use of Translation Editing and Quality Check (TEQC) software (Estey and Meertens, 1999). Bilich and Larson (2007) indicated certain limitations associated with MP1 and MP2 derived from TEQC software to map multipath environments. One limitation is the use of Root Mean Square (RMS) error values to represent multipath. In the current work the RMS values that resulted are shown in

Table 1. The RMS error values lack spatial components and as a result, multipath around the GPS station cannot be visualised. The second limitation involves MP1 and MP2 being a measure of pseudo-range precision, which is highly dependent on smoothing algorithms, the antenna used, and firmware changes of the receiver (Ray and Senior, 2003). A good example to visualise multipath is given by Ogaja and Hedfors (2007), where they developed a MATLAB program for creating colourised polar maps of high-frequency multipath using TEQC report files.

Table 1. Averaged Root Mean Square (RMS) calculated for all the satellites in view during specific days of the experiment. Each observation lasted for 24 hours.

\begin{tabular}{ccc}
\hline \multicolumn{3}{c}{ Pseudo-range multipath error } \\
\hline Day Number & MP1 (m) & MP2 (m) \\
\hline 254 & 0.295173 & 0.298755 \\
255 & 0.298189 & 0.298982 \\
256 & 0.298902 & 0.300827 \\
257 & 0.301725 & 0.294977 \\
258 & 0.299806 & 0.301071 \\
259 & 0.31159 & 0.303643 \\
260 & 0.300505 & 0.300962 \\
261 & 0.300956 & 0.296886 \\
262 & 0.290297 & 0.294945 \\
263 & 0.290814 & 0.288795 \\
264 & 0.292615 & 0.292331 \\
\hline
\end{tabular}


Bilich and Larson (2007) introduced a method of extracting multipath from Signal to Noise Ratio (SNR) data that is reported in the standard RINEX file format, where SN1 and SN2 SNR data represents raw signal strength values as given by the receiver for the L1 and L2 phase observations. In the multipath environment, the SNR values measured by the tracking algorithm are a composite of direct and indirect signals. Equation [1] gives SNR (in an environment where only one indirect signal is introduced) as a function of multipath amplitude $A_{m}$, direct amplitude $A_{d}$ and multipath relative phase $\psi$. Refer to Bilich and Larson (2007) for the derivation of Equation 1. However, this method works well when high data rates (1 s sampling interval) are available.

$$
S N R^{2} \equiv A_{c}^{2}=A_{d}^{2}+A_{m}^{2}+2 A_{d} A_{m} \cos \psi .
$$

We make use of the MP1 and MP2 values derived from the TEQC software to further enhance multipath mapping by creating a gridded sky plot from the derived multipath values using a linear interpolation method and computing the wavelet power spectrum of the multipath time-series data to understand the magnitude in relation to the spatial location of the objects inducing multipath.

Multipath signals from GNSS are non-stationary signals since the frequency of the signals varies in space and time. This makes Wavelet Analysis (WA) suitable for studying multipath behaviour over time as compared to the traditional Fourier Transform (FT) method. We use the Morlet wavelet, which is given by

$$
\psi_{o}(\eta)=\pi^{-1 / 4} \exp \left(i \omega_{o} \eta\right) \exp \left(-\eta^{2} / 2\right) .
$$

Where $\eta$ is the time and $\omega_{o}$ is the wavenumber (Torrence and Compo, 1998). To construct a picture depicting the amplitude of any feature with its variations over time and scale, a continuous wavelet transform $\left(W_{n}\right)$ defined as the convolution of $x_{n^{\prime}}$ (MP1 and MP2) time series with a scaled and translated version of $\psi$ is used to derive the wavelet power spectrum, which is given in Equation 1,

$$
W_{n}(s)=\sum_{n^{\prime}=0}^{N-1} x_{n^{\prime}} \psi *\left[\frac{\left(n^{\prime}-n\right) \delta t}{s}\right]
$$

Where $N$ is the number of points in the time series at sampling time interval $\delta t$ (30 seconds) and $(*)$ represents the complex conjugate (Torrence and Compo, 1998). 
The standard deviation is calculated for each pixel of the linear interpolated multipath matrix as given by Equation 4,

$$
\sigma=\sqrt{\frac{1}{n} \sum_{i=1}^{n}\left(x_{i}-\bar{x}\right)^{2}} .
$$

Where $x_{i}$ is the linear interpolated MP1 or MP2 values and $\bar{x}$ is the mean value of MP1 or MP2. These data are projected to azimuth $(\varphi)$ and elevation $(\theta)$ values as a function of time i.e. $t \rightarrow \varphi(t), \theta(t)$. A higher value of $\sigma$ will indicate areas that have high noise (e.g. trees, variation in moisture content) and low values indicate a uniform medium over time (e.g. clear sky or dry bare soil or less vegetated areas). However, it must be noted that this method is limited to the time span of the data since $\sigma$ will change depending on the number of observations available. Therefore, multipath analysis using this method requires long timeseries (e.g. more than a month) to capture all the seasonal variations due to changes in seasons, which will affect e.g. growth patterns of the trees and this will affect reflected patterns of the L1 and L2 signals.

\section{Results and Discussion}

The GPS satellites provide many data points, but these points are only concentrated at specific satellite tracks and some parts of the sky are not fully covered (e.g. areas between $90^{\circ}$ and $270^{\circ}$ azimuths have a poor coverage in Figure 3 ). The gridded plots in Figure $3(\mathrm{C}$, $\mathrm{B}$ and $\mathrm{D})$ provide a complete picture of the distribution of multipath as well as signal-to-noise ratio (SNR) around the GPS station. As illustrated in Figure 3, most multipath errors occur below $30^{\circ}$ elevation angle and this is associated with low SNR ratio values. Radio signals from satellites at low elevation angles travel longer in the atmosphere (i.e. troposphere) than when the satellites are at high elevations (Degnan, 1993). Hence, the observations below $30^{\circ}$ elevations are noisier as compared to those at a higher elevation. The "spaghetti" features in MP1 and MP2 plots of Figure 3 at both low and high elevations indicating a variable atmosphere and these features could be attributed to clouds or cirrus clouds that vary in space and time. Infrared satellite cloud cover data indicated variable cloud cover during the time of the experiment. The data for the cloud cover can be retrieved from (http://www2.sat24.com/history.aspx? culture=en) for the given dates of the experiment. 


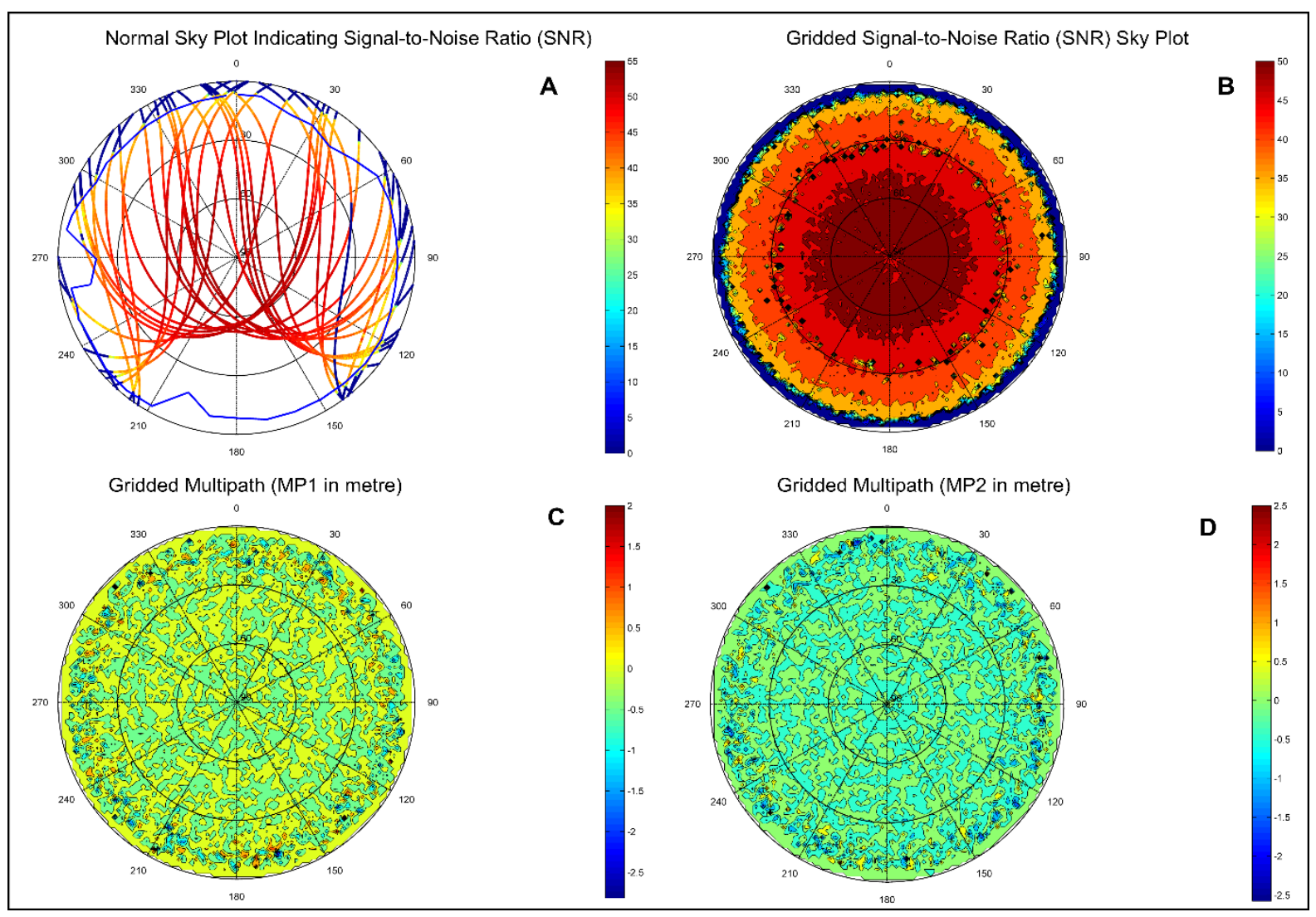

Figure 3. Enhanced visualisation of multipath and SNR data, the normal sky plot indicate satellite tracks across the sky while gridded plots indicate a complete picture distribution of multipath around the GPS station.

Satellite number 17 was selected for the wavelet analysis since the satellite tracks were at both low and high elevation angles. Figure 4 and Figure 5 illustrate the wavelet power spectrum for MP1 and MP2 signals respectively. Two different clusters of peaks are evident at 0.001 and 0.005 frequencies for MP1 in Figure 4, which corresponds to 21 min and 4 min cycles, respectively. The MP1 signal has a strong cycle at 4 min compared to the MP1 signal in Figure 5, which reveals a strong cycle at $21 \mathrm{~min}$ and a weaker cycle at $4 \mathrm{~min}$. The two signals appear to respond differently when reflected by the same features and this is largely due to the fact that MP1 and MP2 are derived from different radio wavelengths where L1= 19 $\mathrm{cm}$ and $\mathrm{L} 2=24.4 \mathrm{~cm}$, respectively. 

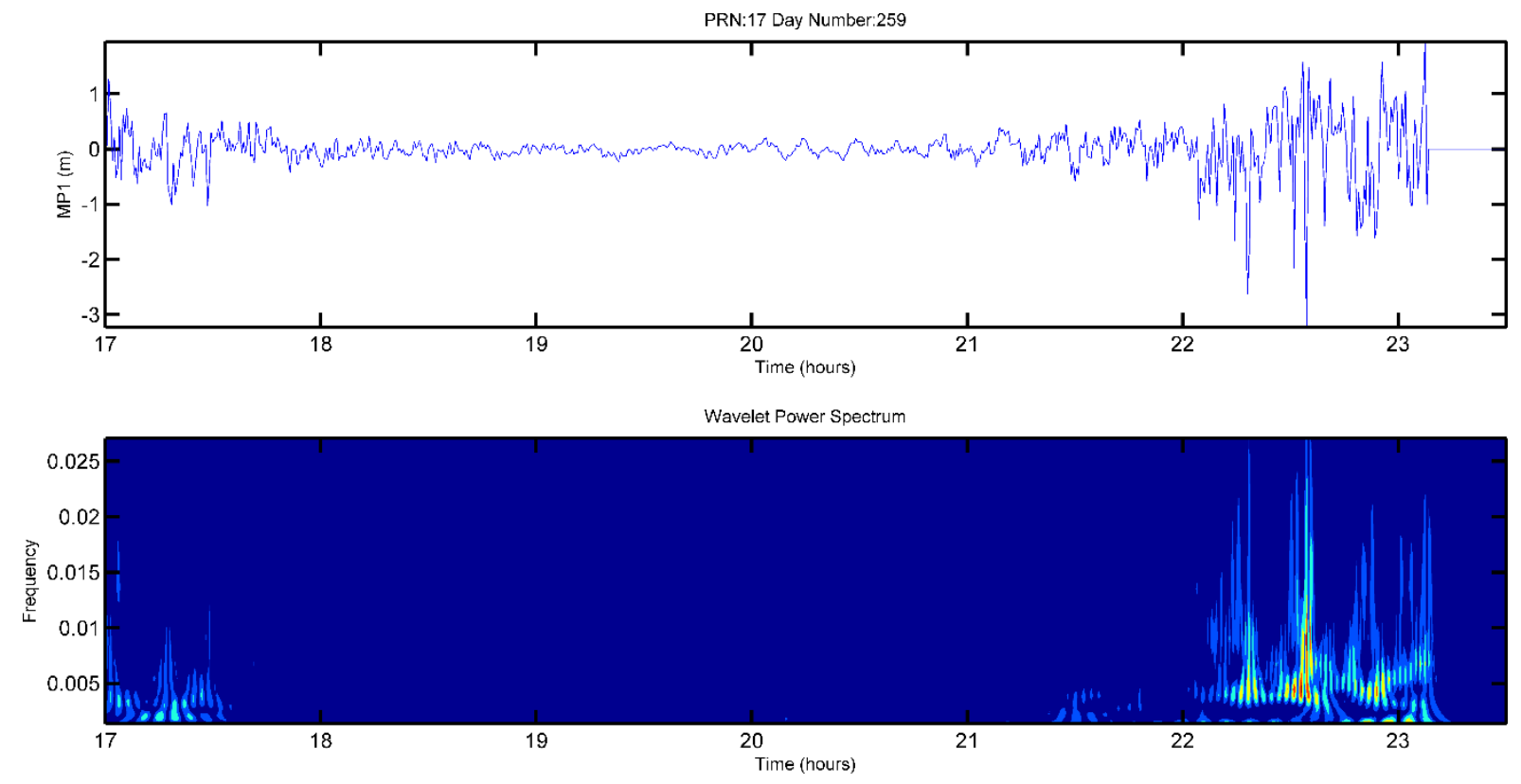

Figure 4. The multipath signals derived from the L1 band and its associated wavelet power spectrum
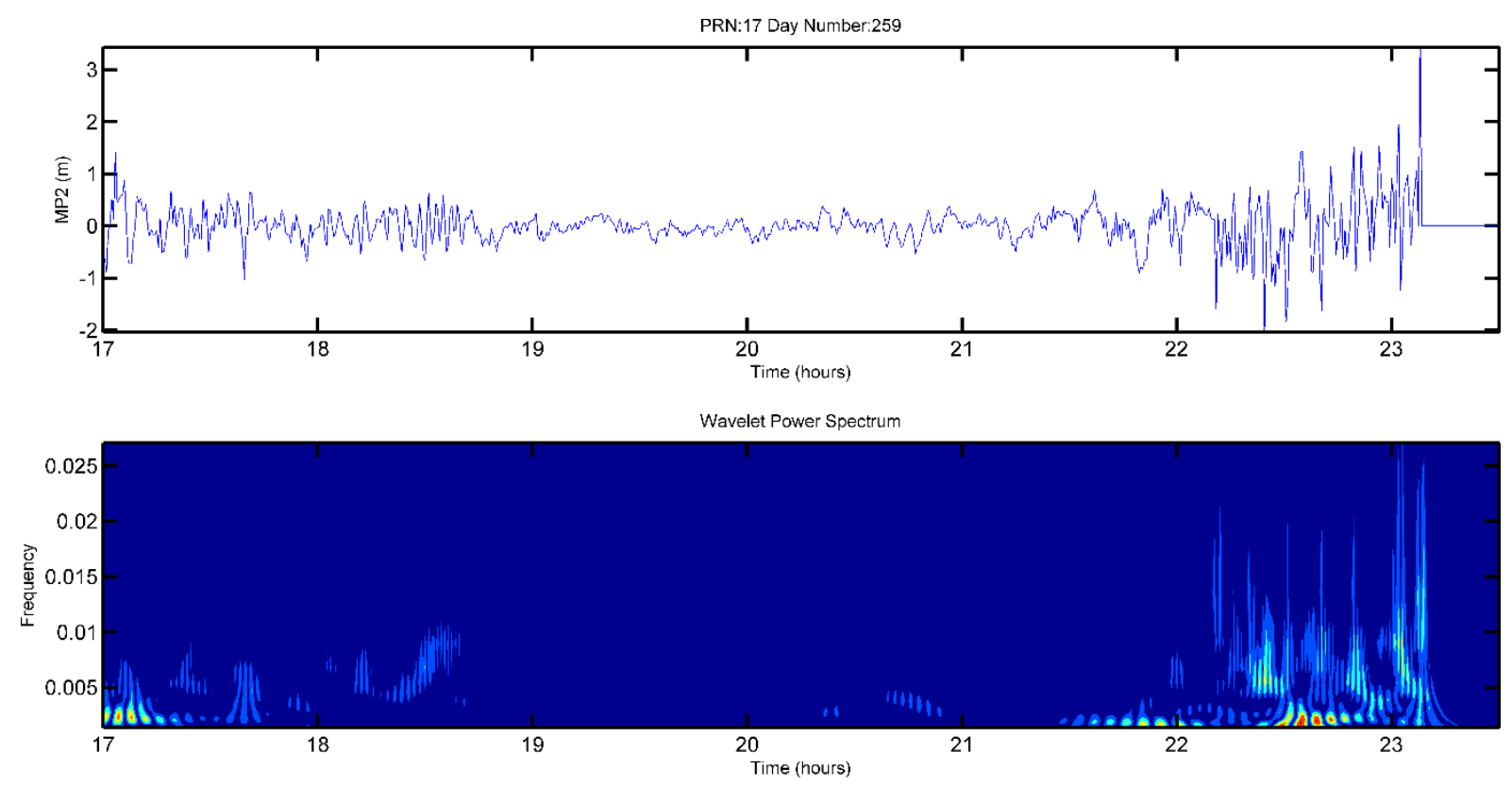

Figure 5. The multipath signals derived from the L2 band and its associated wavelet power spectrum

The standard deviation maps in Figure 6 depict variations of the multipath over the 10 days observation period. Areas on the sky plot with low standard deviation indicate either stationary reflective objects such as ground reflections at low elevation angles $\left(<30^{\circ}\right)$ or atmospheric effects, which is expected to have very little variations at high elevation angles $\left(>30^{\circ}\right)$. Areas with high standard deviation will only occur at lower elevation angles, objects such as shrubs/trees will introduce multipath with different magnitudes resulting in high standard deviation values on the map. Both MP1 and MP2 maps indicate areas of high 
standard deviations and low standard deviations, low standard deviation areas are therefore attributed to reflections due to bare soil and areas with less vegetation, and those of high standard deviation are attributed to reflection due to shrubs as they can be seen from the image mosaic of $360^{\circ}$ view of the area.

There is very little that can be done in terms of physically removing shrubs to minimise multipath. Most applications such as positioning depend on receivers that can filter GPS noise "multipath" at low elevations such as adaptive filters (Yedukondalu et al., 2011). However, this approach is limited to visualisation and the spatial distribution of the source of the multipath. In other applications such as GNSS-Reflectometry, visualisation of multipath is important so as to understand the source of signals that might be related to e.g., vegetation monitoring using GPS (Chew et al., 2015). In the case of positioning applications where time accuracy received from the GPS antenna depends on the position stability of the antenna, an observational strategy will be employed i.e., observing only satellites above $15^{\circ}-20^{\circ}$ elevation angles to minimise multipath.

More improvements still need to be made in this approach of mapping multipath to allow for mapping the distance of reflecting features on the sky-plot. The current approach does not provide exact distances from the GPS station to the reflection points on the ground. This is important in a preliminary site survey using GPS as objects that can result in significant multipath can be mapped and removed before permanent installation of the GPS antenna. For example, similar studies have developed strategies to mitigate multipath in GNSS applications. Zhong et al., (2007) developed an adaptive wavelet transform based on crossvalidation method with application to GPS multipath mitigation. The best results indicated that this method can improve GPS accuracy from 55 to 78\%. Weinbach et al., (2009) used a least mean square adaptive filter, which takes advantage of repetitive nature of multipath errors. This method uses multipath data from the previous data as a reference input to identify common error components in the following day. The choice of suitable sites remains an important factor in minimising multipath errors in GNSS applications. 


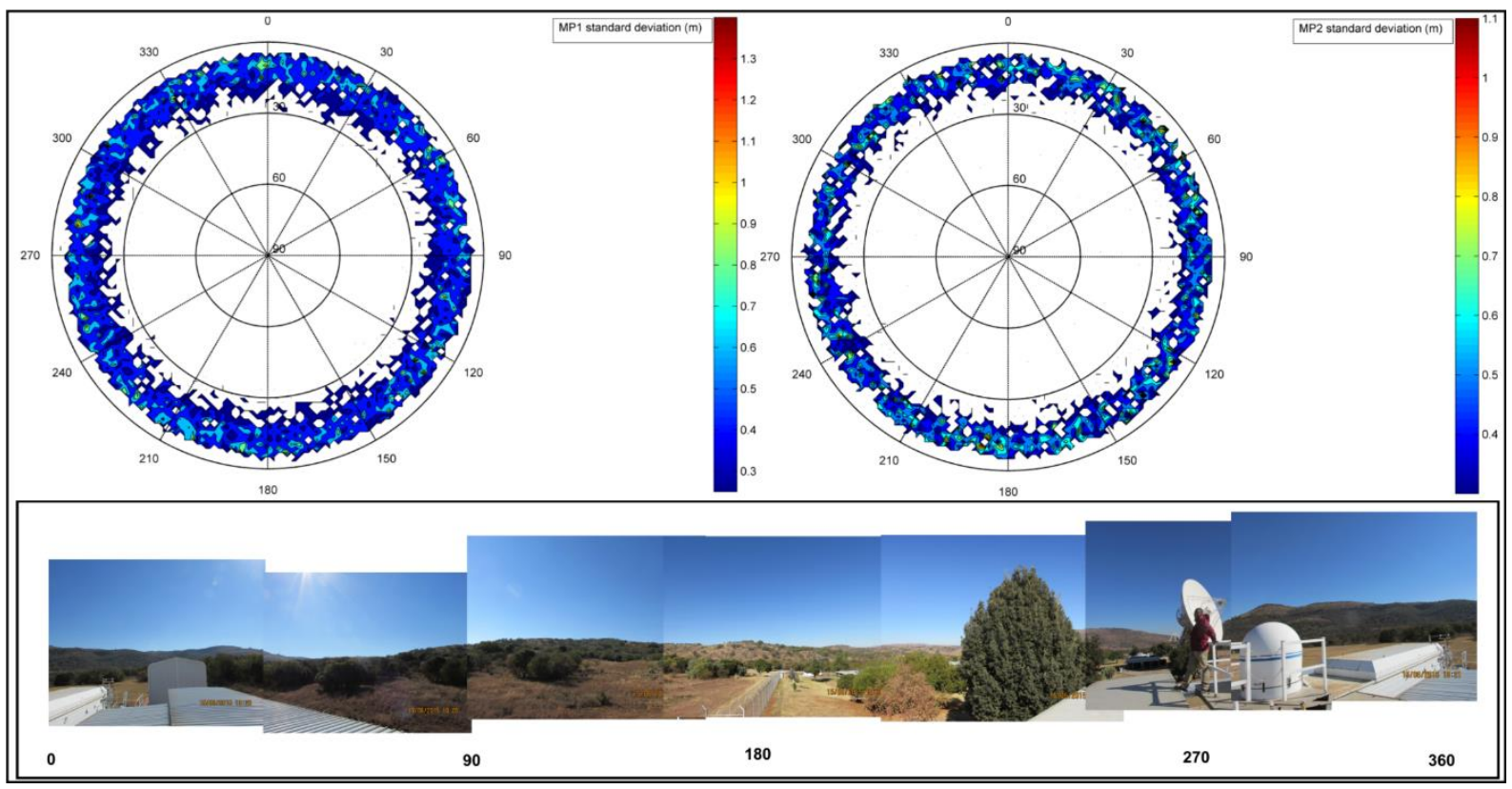

Figure 6. Standard deviation maps of MP1 and MP2 as viewed from the GPS timing antenna derived from 10 days of observations. The mosaics of pictures depict the actual site in $360^{\circ}$ view.

\section{Conclusion}

Mapping multipath from GNSS signals is a difficult task given the many variables that change over a short and long period of time. These variables include moisture content of the soil or vegetation, canopy structure and clouds. The technique of multipath mapping can be very useful in preliminary site investigation for geodetic applications such as the installation of a new GNSS base station for timing or positional applications. We used RINEX observations for 10 days to map spatial location of multipath and its variation over time to aid in understanding the distribution of multipath before an installation of the timing antenna at HartRAO for LLR timing reference purposes.

High-resolution time-frequency wavelet analysis demonstrates that the MP1 and MP2 signals peak at different frequencies with different intensities when reflected by the same feature. This analysis allows for extraction of information such as the time of day that the GNSS station receive maximum multipath (altered by changing geometry of the satellites constellation), how long this condition lasts, and whether an observational strategy can be implemented. Through standard deviation maps, we conclude that the multipath on the site is mainly due to reflections from the mountains surrounding the geodetic site and that the site offers clear sky view of the satellites with minimal multipath from $30^{\circ}$ and above in elevation. The high standard deviation is attributed to reflections due to shrubs, vegetation and bare moist soil around the site. An observational strategy limiting the use of satellite below an elevation of $15^{\circ}$ to $20^{\circ}$ will be implemented as this will offer minimal multipath and still enable adequate satellite visibility. 


\section{Acknowledgements}

This research was financially supported by the National Research Foundation (NRF) and the Department of Science and Technology. We are grateful to the anonymous reviewers for their comments that substantially improved this manuscript.

\section{References}

Allan DW \& Weiss MA 1980, 'Accurate time and frequency transfer during common-view of a GPS satellite', Proc. 43th Ann. Freq. Control Symposium, USAEADCOM, Ft. Monmouth, NJ 07703, National Bureau of Standards Boulder, Colorado, pp. 334-346.

Bilich A \& Larson KM 2007, 'Mapping the GPS multipath environment using the signal-to-noise ratio (SNR)', Radio Science, vol. 42(RS6003), pp. 1-16, dio:1029/2007RS003652.

Böhm J, Möller G, Schindelegger M, Pain G \& Weber R 2015, 'Development of an improved empirical model for slant delays in the troposphere (GPT2w) ', GPS Solutions, vol. 19, no. 3, pp. 433-441, doi: 10.1007/s10291-014-0403-7.

Botteron C, Dawes N, Leclère J, Skaloud J, Weijs SV \& Farine PA 2013, 'Soil Moisture \& Snow Properties Determination with GNSS in Alpine Environments: Challenges, Status, and Perspectives', Remote Sensing, vol. 5, no. 7, pp. 3516-3543.

Chew C, Small EE, Larson KM \& Zavorotny V 2015, 'Vegetation Sensing Using GPS interferometric reflectometry: Theoretical Effects of Canopy Parametres on Signal to Noise Ratio Data', IEEE Transaction on Geoscience and Remote Sensing, vol. 53, no. 5, pp. 2755-2763, doi:10.1109/TGRS.2014.2364513.

Closas P, Fernández-Prades C \& Fernandez-Rubio JA 2009, 'A Bayesian Approach to Multipath Mitigation in GNSS Receivers', IEEE journal of selected topics in signal processing, vol. 4, no. 3, pp. 695-706, doi:10.1109/JSTSP.2009.2023831.

Degnan J 1993, 'Millimetre accuracy satellite laser ranging', in DE Smith, DL Turcotte (eds.), Contributions of space geodesy to geodynamics: Crustal dynamics. Geodynamics Series 25, American Geophysical Union, Washington DC, pp. 133-162.

Estey LH \& Meertens CM 1999, 'TEQC: The multipurpose toolkit for GPS/GLONASS data', GPS solutions, vol. 3, no. 1, pp. 42-49.

Giffard R 1999, Estimation of GPS ionospheric delay using L1 code and carrier phase observables, in McCarthy D \& Fruehauf H (eds.), $31^{\text {st }}$ Annual Precise Time and Time Interval (PTTI) Meeting, California, December 1999, pp. 405-417, Precise Time and Time Interval.

Gurtner W 2007, 'RINEX: The Receiver Independent Exchange Format'. Version 3.00, viewed 06 February 2016, <Whttps://igscb.jpl.nasa.gov/igscb/data/format/rinex300.pdf>. 
Larson KM, Braun JJ, SmallEE, Zavorotny VU, Gutmann ED \& Bilich AL 2010, 'GPS Multipath and Its Relation to Near-Surface Soil Moisture Content', IEEE Journal of Selected Topics in Applied Earth Observations and Remote Sensing, vol. 3, no. 1, pp. 91-99.

Mironov VL \& Muzalecsjiy KV 2012, The new algorithm for retrieval of soil moisture and surface roughness from GNSS Reflectometry'. Geoscience and Remote Sensing Symposium (IGARSS), 2012 IEEE International, pp. 7530-7532, doi: 10.1109/IGARSS.2012.6351889.

Munghemezulu C, Combrinck L \& Botai OJ 2016, 'A review of the lunar laser ranging technique and contribution of timing systems', South Africa Journal of Science, vol. 112, no. 3(4), pp. 50-59.

Ogaja C \& Hedfors J 2007, 'TEQC multipath metrics in MATLAB', GPS Solutions, vol. 11, no. 3, pp. 215-222.

Njoku EG \& Entekhabi D 1996, 'Passive microwave remote sensing of soil moisture', Journal of hydrology, vol. 184, no. 1, pp.101-129, doi:10.1016/0022-1694(95)02970-2.

Pullen S, Park YS \& Enge P 2009, 'Impact and mitigation of ionospheric anomalies on ground-based augmentation of GNSS', Radio Science, vol. 44, no. 1 (RS0A21), pp. 1-10.

Ray J \& Senior K 2003, 'Geodetic techniques for time and frequency comparisons using GPS phase and code measurements', Metrologia, vol. 40, pp. 215-232, doi:10.1088/0026-1394/42/4/005.

Rao GSB, Kumar GS \& Kumar MNVSS 2013, 'GPS signal Rician fading model for precise navigation in urban environment', Indian Journal of Radio and Space Physics, vol. 42, June 2013, pp. 192-196.

Ratcliffe JA 1972. Introduction to the ionosphere and magnetosphere. Cambridge University Press, Great Britain.

Schubert FM, Fleury BH, Robertson P, Prieto-Cerdeirai R, Steingass A \& Lehner A 2010, 'Modeling of multipath propagation components caused by trees and forests'. In Proceedings of the Fourth European Conference on Antennas and Propagation, IEEE., pp. 1-5.

Tregoning P, Boers R, O’Brien D \& Hendy M 1998, 'Accuracy of absolute precipitable water vapour estimates from GPS observations', Journal of Geophysical Research, vol. 103, no. D22, pp. 701728.

Torrence C \& Compo GP 1998, 'A practical guide to wavelet Analysis', Bulletin of the American Meteorological Society, vol. 79, pp. 61-78.

Tregoning P, Boers R, O’Brien D \& Hendy M 1998, 'Accuracy of absolute precipitable water vapour estimates from GPS observations', Journal of Geophysical Research, vol. 103, no. D22, pp. 701728, doi:10.1029/98JD02516 
Wan W, Larson KM, Small EE, Chew CC \& Braun JJ 2015, 'Using geodetic GPS receivers to measure vegetation water content', GPS Solutions, vol. 19, pp. 237-248, doi: 10.1007/s10291-0140383-7.

Weinbach U, Raziq N \& Collier P 2009, 'Mitigation of periodic GPS multipath errors using a normalised least mean square adaptive filter', Journal of Spatial Science, vol. 54, no. 1, pp. 1-13.

Yedukondalu K, Sarma AD \& Srinivas VS 2011, 'Estimation and mitigation of GPS multipath interference using Adaptive Filtering', Progress in Electromagnetics Research M, vol. 21, pp. 133148.

Zavorotny VU, Larson KM, Braun JJ, Small EE, Gutmann ED \& Bilich AL 2010, 'A physical model for GPS multipath caused by land reflections: Toward bare soil moisture retrievals', IEEE Journal of Selected Topics in Applied Earth Observations and Remote Sensing, vol. 3, no. 1, pp. 100-110. doi: 10.1109/JSTARS.2009.2033608.

Zhong P, Ding XL, Zheng DW, Chen W \& Huang DF 2008, 'Adaptive wavelet transform based on cross-validation method and its application to GPS multipath mitigation', GPS Solutions, vol. 12, no. 2, pp. 109-117. 